\title{
PROGRAMAS DE QUALIDADE NO ATENDIMENTO AO TRAUMA
}

\author{
QUALITY PROGRAMS ON TRAUMA CARE
}

Gustavo Pereira Fraga

Docente. Disciplina de Cirurgia do Trauma. Departamento de Cirurgia. Faculdade de Ciências Médicas - UNICAMP. Pós-Doutorado na Universidade da Califórnia, San Diego, com bolsa FAPESP.

CorrespondênciA: Gustavo Pereira Fraga. Avenida Coronel Silva Telles, 211 apto. 3. Cambuí. 13024-000 - Campinas - SP. e-mail: fragagp@uol.com.br

Fraga GP. Programas de qualidade no atendimento ao trauma. Medicina (Ribeirão Preto) 2007; 40 (3): 321-8, jul./set.

RESUMO: O trauma é um problema de saúde pública de grandes proporções. É a principal causa de óbito na população jovem. Pesquisas mostram um grande número de pessoas morrendo de lesões que poderiam ser prevenidas. Estes óbitos evitáveis podem diminuir com meIhorias na organização e planejamento dos serviços de trauma. Uma das medidas administrativas utilizadas para melhorar a assistência ao trauma são programas de controle de qualidade conhecidos como auditoria médica ou processos contínuos de melhora da qualidade. O programa de melhoria de qualidade é um método contínuo de aperfeiçoar a assistência médica através da monitoração dos elementos de diagnóstico, tratamento e evolução dos pacientes. O objetivo deste controle de qualidade é assegurar que os sistemas e centros de trauma determinem sistematicamente processos de monitoração, análise e controle dos resultados com o objetivo final de melhorar a evolução dos pacientes tratados. Inúmeras técnicas têm sido utilizadas para desenvolver este processo: reuniões de morbimortalidade, estudos de óbitos evitáveis, registros institucionais, protocolos, auditorias, escores de risco e avaliação do prognóstico. No Brasil existem apenas informações rudimentares de mortalidade devido à falta de programas para coletar dados e avaliar as informações relacionadas ao trauma. Este artigo apresentará o estado atual dos programas de controle de qualidade para avaliar a assistência às vítimas de trauma.

Descritores: Trauma. Centros de Traumatologia; organização \& administração. Controle de Qualidade. Registro Médico Coordenado. Mortalidade.

\section{1- INTRODUÇÃO}

O trauma é um crescente problema de saúde em todo o mundo. Estima-se que, diariamente, 16 mil pessoas morrem vítimas de causas externas, e para cada vítima fatal, ocorrem outros milhares de traumatismos, muitos destes com seqüelas permanentes. ${ }^{1}$ Entre as causas de trauma, incluem-se os acidentes e a violência, que configuram um conjunto de agravos à saúde, que podem ou não levar a óbito, no qual fazem parte as causas ditas acidentais e as intencionais. ${ }^{2}$ No Brasil, em 2004, as causas externas foram responsáveis por 127.470 óbitos. $^{3}$
$\mathrm{Na}$ abordagem do tema programas de qualidade no atendimento ao traumatizado é obrigatório a pesquisa na literatura internacional, uma vez que os países desenvolvidos são pioneiros no assunto, vêm estudando este tema há algumas décadas e geralmente têm recursos financeiros mínimos para estruturação do atendimento às urgências e emergências. Define-se como programa de qualidade os métodos e processos criados para monitorar continuamente os elementos de diagnóstico, tratamento e evolução das vítimas. ${ }^{1,4,5,6}$

A exigência de uma abordagem adequada para tratar um paciente, tal como uma auditoria médica, 
atualmente é extrapolada para o coletivo do sistema de saúde, de modo contínuo, organizado, ético e reservado, para se avaliar a eficácia, custos e resultados da prática médica inserida no tratamento do traumatizado, baseado em evidências e com o objetivo prioritário de melhorar a assistência.

Tais programas de qualidade são imprescindíveis em países que têm estruturado o sistema de atendimento ao trauma, uma vez que fazem parte do processo de educação, capacitação profissional, direcionamento de recursos, estabelecimento de prioridades e até mesmo compromisso social das pessoas envolvidas, nas diferentes fases. Seria como um exercício de cidadania do profissional de saúde, especificamente aquele envolvido num dos elos da abordagem da doença trauma, mostrando os indicadores para que vários setores da sociedade, como educação, cultura, planejamento, legislação, entre outros, se engajem no combate a esta doença.

Portanto, para discutir sobre programas de qualidade se faz necessário que os envolvidos tenham maturidade, ética, autocrítica e interesse contínuo em aperfeiçoamentos e melhorias. No Brasil, este assunto é relativamente novo. ${ }^{6}$ Para um adequado entendimento, é importante que se tenha noção de conceitos como: sistemas e centros de trauma, e a diferença entre ambos (já abordado neste simpósio); índices e escores de trauma; registros de trauma; reuniões de morbimortalidade; óbitos evitáveis; protocolos e condutas em trauma; medicina baseada em evidências; análise criteriosa de resultados e da evolução dos pacientes.

A fim de tornar este artigo didático e agradável para leitura, será apresentado um histórico de como estes programas de qualidade têm sido implantados em países desenvolvidos, com extensão para outros em desenvolvimento. Finalizando, será feita uma análise crítica da situação dos programas de qualidade no atendimento ao traumatizado no Brasil, expressando comentários do autor que poderão ser úteis para aqueles que têm esperança e acreditam num futuro melhor ao se abordar esta doença multi-sistêmica e multifatorial, que é o trauma.

\section{2- HISTÓRICO DOS PROGRAMAS DE QUA- LIDADE}

Os programas de qualidade no atendimento ao trauma têm uma ligação direta com o Colégio Americano de Cirurgiões (American College of Surgeons - ACS), fundado em 1913, com os princípios de me- lhorar a assistência de pacientes cirúrgicos e a educação e capacitação dos cirurgiões. ${ }^{5}$ Em 1922 foi criado o Comitê de Trauma (Committee on Trauma - COT) do ACS, o mais antigo comitê desta instituição, reconhecendo o trauma como uma doença, e focado no desenvolvimento de ações para prevenção e tratamento das vítimas. ${ }^{5}$

A Academia de Ciências Norte-Americana publicou um documento, em 1966, considerando o trauma como a "doença negligenciada da sociedade moderna", chamando a atenção para a necessidade da implementação de programas e ações no sentido de reduzir a morbimortalidade por causas externas. Mas foi em 1976 que o COT - ACS publicou o primeiro protocolo com diretrizes e categorização dos hospitais para atendimento das vítimas de trauma, definindo o conceito de centros de trauma. ${ }^{5,7}$ Este livro contendo os recursos para melhorar a assistência aos pacientes traumatizados foi revisado e novamente publicado em 1990, 1993 e 2006, sendo que esta última versão é mais ampla, reforçando a necessidade do desenvolvimento de sistemas de trauma, ao invés de apenas centros de trauma, de modo organizado, numa determinada região, integrando o pré-hospitalar, os recursos hospitalares e estabelecendo a importância em desenvolver o controle de qualidade e a auditoria dos resultados. ${ }^{5}$

O curso de Suporte Avançado de Vida no Trauma (Advanced Trauma Life Support - ATLS ${ }^{\circledR}$ ) foi criado pelo ACS em $1978 .{ }^{8}$ A implantação do ATLS ${ }^{\circledR}$ tem permitido um preparo mais adequado dos médicos a fim de atuarem na chamada "hora de ouro", onde o diagnóstico e a terapêutica precoces, na primeira hora de atendimento ao traumatizado, podem melhorar a sobrevida. Este curso já se propagou para 47 países, permitindo o treinamento de aproximadamente 500.000 profissionais. Na literatura, há vários artigos apontando uma redução nas taxas de mortalidade em até $50 \%$, após a implantação do $\operatorname{ATLS}^{\circledR}{ }^{9,10}$

Um dos problemas na abordagem do paciente traumatizado é que, até mesmo numa pequena comunidade, grupos de pessoas lesadas diferem entre si quanto à natureza e gravidade das lesões. Esta heterogeneidade e a dificuldade em ajustar tais variações têm estimulado vários estudos científicos envolvendo as pessoas vítimas de traumatismo. Foram então criados os Índices ou Escores de Trauma, que são valores matemáticos ou estatísticos, quantificados por escores numéricos, que variam de acordo com a gravidade das lesões resultantes do traumatismo. ${ }^{11,12}$ Estes índices são divididos em três grandes grupos: fisiológi- 
cos, anatômicos e mistos. ${ }^{11}$ Alguns índices são muito específicos, porém outros são de aceitação universal, sendo periodicamente revistos e atualizados a fim de se tornarem mais precisos. ${ }^{12}$

A necessidade de se criar um método adequado para quantificar a gravidade das vítimas de trauma fez com que cirurgiões se reunissem, em 1982, a fim de desenvolver um teste que estimasse a probabilidade de sobrevida de um traumatizado. O "Trauma Revised Injury Severity Score (TRISS)" foi o índice adotado e, sob a coordenação do COT - ACS foi desenvolvido um estudo para avaliar os resultados de pacientes traumatizados, denominado "Major Trauma Outcome Study" ou MTOS. ${ }^{13} \mathrm{O}$ objetivo inicial deste estudo era desenvolver normas para o tratamento dos traumatizados que pudessem ser adotadas nos hospitais a fim de garantir uma qualidade de atendimento nos sistemas de urgência. Com isso seria possível aos hospitais compararem seus resultados em grupos de pacientes com a mesma gravidade, e identificar, para posterior análise, os pacientes que morreram inesperadamente, bem como identificar os traumatizados sobreviventes apesar de uma predição para morrer. A identificação destes pacientes, cujos resultados desviem do padrão estabelecido, permite avaliar os óbitos evitáveis ou possivelmente evitáveis, tentando identificar eventuais falhas no diagnóstico, interpretação ou aplicação de técnicas, motivando a equipe médica de urgência à mudança de eventuais condutas. ${ }^{4,13}$

Os índices de trauma, incluindo o TRISS, têm uma série de limitações. Portanto, todo traumatizado que evolui a óbito precisa ter o seu atendimento revisado, promovendo uma auditoria no atendimento, com a participação de equipes multidisciplinares. ${ }^{1,4,5,6}$

Em 1987 o ACS publicou um documento sobre Sistemas de Trauma, com o objetivo de estabelecer diretrizes para a implantação e desenvolvimento destes sistemas. ${ }^{14}$ Neste mesmo ano, o Colégio Americano de Médicos Emergencistas (American College of Emergency Physicians) também editou um artigo com diretrizes e protocolos para a organização de sistemas e atenção no atendimento ao traumatizado. ${ }^{15}$ É importante ressaltar que a implantação destes sistemas de trauma regionalizados ocasionou uma redução significativa da mortalidade em traumatizados. ${ }^{7,16}$ Nos EUA, apenas $50 \%$ dos estados possui sistema de trauma organizado. ${ }^{7}$ Em estudo recente, Nathens et al. ${ }^{16}$ compararam a mortalidade de traumatizados através de registros em estados norte-americanos com e sem sistemas de trauma. Estes autores observaram uma mortalidade 9\% menor nos estados com sistema de trauma e, analisando apenas os acidentes por veículo motorizado, a redução da mortalidade foi de $17 \%$. Biffl et al. ${ }^{17}$ avaliaram vítimas de trauma atendidas num centro de trauma nível I, o único na Ilha de Rhode, no norte dos EUA, em dois períodos distintos: 1991 a 1993, e 1999 a 2001. Os autores observaram uma maior mortalidade $(6,8 \%$ versus $4,4 \%)$ no período mais recente, porém eram pacientes com idade mais avançada e com traumas mais graves, chamando a atenção para necessidade de um processo contínuo de monitoração dos casos. ${ }^{17} \mathrm{Em}$ artigo recente, Celso et al. ${ }^{18}$ fizeram uma revisão de 14 artigos publicados na literatura sobre resultados após a implantação de um sistema de trauma, e através de meta-análise, observaram uma redução da mortalidade, em média, de 15\%.

Em 2004, a Associação Internacional de Cirurgia (International Society of Surgery), a Associação Internacional de Cirurgia do Trauma e Terapia Intensiva (International Association for The Surgery of Trauma and Surgical Intensive Care - IATSIC) e a Organização Mundial de Saúde (World Health Organization ou OMS), após a constituição de um grupo de trabalho que atuou conjuntamente durante alguns anos, publicaram um livro com as diretrizes para assistência ao trauma (Guidelines for essential trauma care). ${ }^{1}$ Este livro foi desenvolvido com o objetivo de promover melhorarias no atendimento às vítimas de trauma, principalmente em países em desenvolvimento. É voltado para profissionais que atuam no planejamento, organização de sistemas e atendimento dos pacientes, além de sociedades médicas de especialistas e instituições de ensino, a fim de divulgarem e buscarem melhores condições para assistência ao traumatizado. ${ }^{1}$ A fim de promover uma melhor qualidade no atendimento, alguns elementos são importantes: recursos humanos, com formação, capacitação e treinamento continuado dos profissionais; recursos físicos, com infra-estrutura adequada, equipamentos e investimentos; e organização e administração do processo, com auditoria nos resultados. A Tabela I cita os itens que fazem parte deste projeto, onde estão incluídos programas de qualidade no atendimento ao trauma.

\section{3- CONTROLE DE QUALIDADE NO TRAU- MA}

A atenção ao traumatizado geralmente é feita através de sistemas integrados de atendimento, que 
Tabela I: Métodos para promoverem melhorias na assistência ao traumatizado

- Treinamento de médicos e outros profissionais para atendimento ao traumatizado;

- Organização das equipes de trauma e de atendimento inicial, em serviços com diferentes complexidades;

- Inspeção nos hospitais e serviços de atendimento ao traumatizado;

- Integração entre os sistemas pré-hospitalar, hospitalar e reabilitação;

- Interação e integração com autoridades e instituições governamentais (Ministério da Saúde);

- Programas de melhoria na qualidade da assistência;

- Progresso nos registros e resultados.

devem incluir: prevenção, atendimento pré-hospitalar, atendimento hospitalar e reabilitação. Para se implantar um programa de qualidade em trauma, alguns pontos são essenciais: determinação prévia dos padrões de qualidade; definição dos critérios para monitoração dos resultados; anotação e registro dos dados; análise e revisão dos erros e problemas identificados. ${ }^{1,6}$ Os padrões de qualidade dependerão diretamente dos recursos humanos e materiais disponíveis.

Os itens mais importantes para implementação destes programas de qualidade serão discutidos a seguir.

\section{1- Registro de trauma}

O registro de trauma é um banco de dados específico para esta doença composto de uma série de informações uniformes que descrevem características demográficas e epidemiológicas, o mecanismo de trauma, informações de atendimento pré-hospitalar, diagnósticos, tratamentos efetuados, evolução dos pacientes e gastos. ${ }^{5}$ Estes registros devem coletar dados referentes aos traumatismos, classificá-los, definir a gravidade e agrupar as informações para análises individuais e coletivas. Os registros de trauma são imprescindíveis para o direcionamento das decisões e ações pertinentes às vítimas de trauma. Estes registros servem como uma ligação entre o que é preciso ser conhecido sobre as causas e resultados dos traumas, e o desenvolvimento de planos de intervenção local, regional e nacional.
A documentação das informações deve ser realizada em cada serviço que atende ao paciente. Inicialmente, é importante definir os critérios de inclusão e exclusão de pacientes no registro. As informações devem ser coletadas em tempo real, portanto o ideal é que se tenha uma equipe treinada coletando diariamente estas informações. ${ }^{6}$ É necessário um sistema informatizado para armazenamento destes dados, de maneira que as informações possam ser facilmente acessadas e os resultados analisados de maneira prática e rápida, através de computadores. Com isto, é possível a obtenção de informações em um hospital, cidade, estado e até mesmo em todo o país.

Em países desenvolvidos, o sistema de saúde se baseia em informações coletadas em grandes bancos de dados, num processo contínuo de cooperação e coordenação de ações no intuito de melhorar a assistência médica. Nos Estados Unidos da América (EUA), o ACS desenvolveu o "National Trauma Data

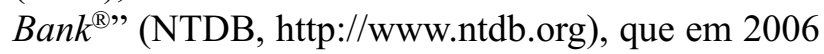
já acumulava cerca de dois milhões de casos coletados ao longo de uma década, com informações de 640 centros de trauma, de 45 estados americanos. ${ }^{19}$ No Canadá, a discussão da necessidade de se implantar um registro nacional de trauma se aprofundou em 1997. ${ }^{20}$ O "Canadian Institute for Health Information" (CIHI) passou a coletar dados no "National Trauma Registry" (NTR, http://www.cihi.ca/ntr) em 2001. No boletim referente a hospitalização de traumatizados em 2005, com análise do período 2002-2004, foram cadastrados 388.904 casos, com os seguintes achados epidemiológicos: predomínio do sexo masculino (53\%); média de idade de 52 anos; quedas como o principal mecanismo de trauma (57\%), seguido de acidentes de veículos motorizados (14\%); média de internação de 10 dias; e mortalidade global de $4 \%{ }^{21}$ $\mathrm{O}$ conhecimento destas informações é fundamental para o mapeamento da doença trauma no país.

$\mathrm{Na}$ Austrália, no estado de Vitória, com população de 4,7 milhões de pessoas (24\% dos habitantes daquele país), um registro de trauma foi implantado em 2002. Em estudo descrevendo os resultados dos dois primeiros anos de funcionamento do registro, quando foram atendidos 2746 pacientes, os autores observaram que $80 \%$ dos traumatizados graves foram tratados em centros de trauma e a mortalidade (intra-hospitalar de aproximadamente 14\%) foi comparável com padrões internacionais. ${ }^{22} \mathrm{O}$ registro de trauma permitiu monitorar os resultados e identificar algumas áreas que precisam ser melhoradas. ${ }^{22}$ 
$\mathrm{Na}$ Itália, o primeiro registro de trauma foi desenvolvido em $2004 .{ }^{23}$ Em outros países em desenvolvimento, registros de trauma começam a ser implantados. Mock et al. ${ }^{24}$ compararam a mortalidade em três grandes cidades com diferentes níveis sócio-econômicos: Kumasi, em Gana (63\% de mortalidade em pacientes com ISS $\geq 9$ ); Monterrey, no México (55\%); e Seattle, nos EUA (35\%). Os autores observaram que nos países menos desenvolvidos a mortalidade era muito maior no pré-hospitalar, identificando que este deva ser o foco inicial para investimentos em Gana e no México. ${ }^{24}$ Isto comprova a importância do registro na promoção do controle e melhora de qualidade.

A precisão no registro das informações é fundamental, principalmente para controle de qualidade. Hlaing et al. ${ }^{25}$, em estudo realizado num centro de trauma nível II nos EUA, observaram que a taxa de erro nas informações variou conforme o dado registrado, sendo em média de 3\%. Erros do tipo duplicidade de informação, registro de códigos errados, falta de anotação cronológica adequada, ou poucos detalhes na evolução do paciente podem ser minimizados com a revisão sistemática por um coordenador do registro, que precisa conhecer muito bem as informações e os erros que podem ocorrer. ${ }^{25}$

\section{2- Desenvolvimento dos sistemas de trauma}

Com a organização de sistemas de trauma tem ocorrido uma redução da mortalidade de 15 a 20\%. ${ }^{1,7,18}$ Já a redução dos óbitos evitáveis chega a 50\%. Em média, apenas $15 \%$ das vítimas de trauma precisam ser atendidas em centros de trauma com maior complexidade, equivalentes, no Brasil, aos hospitais terciários. ${ }^{7}$ A Tabela II cita alguns critérios importantes para a implantação destes sistemas.

Tabela II: Critérios para a implantação de sistemas de trauma.

- Legislação definindo as regras de funcionamento;

- Processo formal de designação e hierarquia dos serviços;

- Padronização dos atendimentos;

- Definição da população e área geográfica de cobertura do atendimento;

- Implantação do pré-hospitalar e critérios de triagem;

- Educação e prevenção;

- Sistemas de controle da qualidade.
Um aspecto importante é o financiamento de recursos nestes sistemas de trauma, que praticamente não existem de maneira organizada em países em desenvolvimento. Após utilizarem o livro Guidelines for essential trauma care $^{1}$ em quatro países (México, Vietnã, Índia e Gana), Mock et al. ${ }^{26}$ observaram que os recursos eram parcialmente adequados ou adequados na maioria dos hospitais visitados, sendo mais escassos em áreas rurais, mas na maioria dos serviços já existiam iniciativas para melhorar a assistência ao traumatizado. Em recente revisão, Mock et al. ${ }^{27}$ chamam a atenção para a necessidade de investimentos em países em desenvolvimento, onde vive a maior parte da população mundial, onde a incidência de traumas é maior e onde ainda há um baixo controle da qualidade da assistência prestada ao traumatizado.

No Brasil, os resultados da implantação de treinamento para os profissionais no Hospital das Clínicas de Ribeirão Preto, com a introdução do curso do ATLS $^{\circledR}$, foram avaliados por Campos Filho ${ }^{10}$, notando-se uma melhora significativa no atendimento ao traumatizado. Em outro estudo realizado nesta cidade com vítimas de acidente de trânsito, Scarpelini et al. ${ }^{28}$ mostraram a importância da implantação de um sistema com atendimento pré-hospitalar na região.

\section{3- Reuniões de morbimortalidade}

Estas reuniões devem abranger discussões relativas aos pacientes que evoluíram com complicações ou a óbito. Vários serviços de cirurgia fazem estas reuniões periodicamente, e o processo de revisão dos casos de trauma, identificando os setores que precisam ser aperfeiçoados, faz parte do controle de qualidade. ${ }^{1,5}$ Nos casos de óbito, é importante a realização de necropsia, principalmente em casos de óbito tardio, a fim de complementar a investigação de cada caso. ${ }^{29}$ Estas reuniões devem ser multidisciplinares, com a participação de profissionais do pré-hospitalar, cirurgiões, ortopedistas, anestesistas, neurocirurgiões, intensivistas, médicos legistas, enfermeiras, e outros profissionais envolvidos no atendimento.

\section{4- Estudo dos óbitos evitáveis}

Neste processo de revisão dos casos de complicações ou óbito, e educação entre os pares, é importante que se classifique o óbito como: inevitável (mesmo nesta situação, em casos selecionados podem ser identificadas medidas para melhorar o atendimento), potencialmente evitável ou francamente evitável. Nas décadas de 60 e 70 vários estudos surgiram analisando conceitos de óbito evitável, avalian- 
do retrospectivamente a mortalidade, muitas vezes de modo empírico, baseado na opinião de profissionais experientes, com taxas de 20 a $40 \%$ de óbitos evitáveis. ${ }^{4}$ Posteriormente, os índices de trauma passaram a ser empregados, para este fim, considerando como evitável o óbito de pacientes com TRISS $\geq 0.50$.

O óbito é sempre um evento sentinela, e todos os casos de óbito devem ser revisados. ${ }^{6}$ A auditoria constante é importante para a redução das taxas de complicações e óbitos evitáveis, promovendo o controle de qualidade. Atualmente, em países em que existem sistemas de trauma, é aceitável uma taxa de óbitos evitáveis de 1 a $2 \%{ }^{7}$

Os casos identificados como desvio do padrão ou erro devem ser discutidos por uma comissão de profissionais de saúde, com experiência em trauma, através de um processo educativo. A identificação de erros implica obrigatoriamente no desenvolvimento de estratégias, principalmente de treinamento da equipe e com caráter instrutivo, para evitar a recorrência destes erros. As medidas adotadas após identificar e corrigir eventuais erros devem ser monitoradas para se avaliar a eficácia do processo. ${ }^{6}$

\section{5- Filtros para auditoria}

O conceito de filtros para auditoria em trauma para identificação de casos com desvio do padrão é baseado no uso de critérios considerados ideais, mas que podem variar, embora se baseiem em consensos. ${ }^{4}$ O próprio ACS definiu no passado alguns filtros que exigiam correção imediata, como exemplo: paciente com escala de coma de Glasgow (ECG) menor que 14 sem tomografia de crânio; paciente com ECG menor ou igual a oito transportado sem uma via aérea definitiva; paciente com hematoma epidural ou subdural submetidos à craniotomia com mais de quatro horas após a admissão, entre outros. A sensibilidade, especificidade e acurácia de cada um destes filtros não é muito confiável. Assim sendo, o próprio ACS deixou de publicar estes filtros na sua mais recente revisão, embora ainda recomendem o uso seletivo. ${ }^{5}$

\section{6- Comitês de revisão de morbimortalidade}

O ideal é que numa determinada região, profissionais de diferentes serviços se reúnam periodicamente para discussão dos casos com resultado fora do padrão determinado. ${ }^{1,5}$ Estes comitês devem seguir os conceitos de medicina baseada em evidências e ter as seguintes características: crítica, porém não destrutiva; honestidade; uniformidade; e sem ligações ou tendências administrativas ou políticas.

\section{7- Fechamento do ciclo}

O controle de qualidade aumenta a medida que uma ação de correção tem o seu resultado comprovando melhora, num processo de avaliação continuada. Cada achado precisa ser documentado e implementado, mostrando uma melhora do controle e da evolução das vítimas. Este ciclo nunca será completado, porque as ações estarão sempre em busca de melhorias, mas a melhor evolução e segurança dos pacientes é uma prova da importância destes programas de qualidade em trauma. ${ }^{5}$

\section{8- Medidas do processo de qualidade}

A importância e o valor da evolução deste processo de controle de qualidade dependem da perspectiva em que o mesmo é avaliado. ${ }^{4,5} \mathrm{O}$ médico visa uma melhor qualidade no tratamento, e o paciente deseja uma completa e rápida recuperação, sem seqüelas. $\mathrm{O}$ administrador visa a redução dos custos. $\mathrm{O}$ valor deste processo pode ser ilustrado pela equação:

Valor $=$ qualidade do processo + qualidade da evolução custos

O valor será maior a medida que melhora a qualidade e evolução dos pacientes, com custos menores.

\section{4- PROGRAMAS DE QUALIDADE NO BRASIL}

No Brasil não existe ainda um sistema organizado de atendimento ao traumatizado, com abrangência nas diferentes fases. O que se observa é o esforço isolado de alguns cidadãos e de setores da sociedade a fim de combater esta doença.

Em nosso meio, há algumas décadas foram criados serviços conceituados com direcionamento para o atendimento de urgência médica, incluindo aí os casos cirúrgicos e vítimas de trauma. Em 1987, foi criada, em São Paulo, a Disciplina de Cirurgia Geral e do Trauma na Faculdade de Medicina da USP e, em Campinas, a Disciplina de Cirurgia do Trauma na Faculdade de Ciências Médicas - UNICAMP. ${ }^{30}$ Desde então, diversos serviços de trauma têm se desenvolvido no Brasil, porém ainda não existe um sistema organizado. Além disso, a inexistência de estudos epidemiológicos e registros dos traumatismos em nível municipal e estadual, a falta de informações referentes ao atendimento pré-hospitalar, e a desintegração entre os hospitais de complexidades diferentes e o Instituto Médico Legal prejudicam um estudo abrangente sobre as causas de óbito, após traumatismo, no Brasil. 
Frente à necessidade de definição, no setor de saúde, de uma política decisiva no sentido da redução da morbimortalidade por acidentes e violências, foi aprovado em 16 de maio de 2001, e publicado, posteriormente, no Diário Oficial da União, uma Portaria visando um conjunto de ações articuladas e sistematizadas a fim de combater esta doença e contribuir para a qualidade de vida da população. ${ }^{2}$ Uma das diretrizes prioritárias estabelecidas foi a monitoração da ocorrência de acidentes e violências, mediante a promoção de auditoria da qualidade de informação dos sistemas, padronização de fichas de atendimento de forma a fornecer o perfil epidemiológico do paciente atendido, criação de um banco de dados visando à união de esforços e à otimização de recursos disponíveis.

Também a formação do cirurgião geral, que geralmente é acionado para o atendimento e tratamento das vítimas de trauma, tem sido bastante limitada, com um programa de dois anos de duração. Muitos optam por complementarem a especialização em diferentes áreas, não tendo interesse e dedicação continuada no tratamento das vítimas de trauma. O Colégio Brasileiro de Cirurgiões (CBC) está estudando soluções para este problema, mas a extinção da residência médica em Cirurgia do Trauma pode estar afastando ainda mais os profissionais das urgências cirúrgicas e trauma.

A Sociedade Brasileira de Atendimento Integrado ao Traumatizado (SBAIT - www.sbait.org.br), atualmente sem reconhecimento junto à Associação Médica Brasileira (AMB) por não representar uma especialidade médica, está aprofundando as discussões para que o cirurgião de urgência e trauma tenha um espaço bem definido para a sua atuação. Este profissional precisa não só ter o domínio de clínica e técnicas cirúrgicas, mas precisa ter conhecimento do que é um sistema de trauma, a epidemiologia do trauma em sua região, a integração com o pré-hospitalar, registros e índices de trauma, e principalmente aprender a desenvolver instrumentos para o controle de qualidade, ainda inexistentes no Brasil.

Nos últimos anos, o movimento de acadêmicos com a formação das Ligas de Trauma, a grande propagação destas pelo país, com ações articuladas e desenvolvimento de recursos humanos com grande interesse por urgências cirúrgicas e trauma, nos enche de esperança de que um futuro melhor pode estar se aproximando das vítimas de trauma e da sociedade brasileira.

\section{5- CONCLUSÕES}

Os programas de controle de qualidade no atendimento ao trauma constituem parte fundamental dos sistemas de trauma em países desenvolvidos. Fazem parte do processo de educação: capacitação, registro dos resultados e auditoria dos tratamentos utilizados. Não tem caráter destrutivo e devem ser baseados em critérios bem definidos a fim de melhorar o atendimento às vítimas. Estes programas de qualidade são continuados e imprescindíveis para a promoção de medidas eficientes de redução de morbimortalidade. No Brasil, existem alguns programas isolados, mas compete a nós, profissionais da área de saúde, que atuamos com urgências cirúrgicas e trauma, a busca das condições para implantar este controle de qualidade.

Fraga GP. Quality programs on trauma care. Medicina (Ribeirão Preto) 2007; 40 (3): 321-8, july/sept.

ABSTRACT: Trauma is a public health problem of vast proportions. It is the leading cause of death in young people. Researches have shown a significant number of persons dying from preventable injuries. The number of these preventable deaths can decrease by improving the organization and planning for trauma care services. One of the key administrative functions used to strengthen trauma care is quality assurance programs, also known as medical audit or continuous quality improvement. Quality improvement is a continuous method of improving medical care by monitoring the elements of diagnosis, treatment and outcome. The goal of quality improvement is to ensure that trauma centers and trauma systems design processes to systematically monitor, analyze, and improve performance with the ultimate intent of improving patient outcomes. A variety of techniques have been used specifically to develop trauma quality improvement: morbidity and mortality conferences, preventable death studies, institutional registries, guidelines, audit filters, risk adjusted scoring, and outcome measures. In Brazil there is only rudimentary information on mortality due to the lack of systematic programs to collect data and information on trauma. This paper will emphasize the current status of quality improvement methodology to assess the care of injured patients.

Keywords: Trauma. Trauma Centers: organization \& administration. Quality Control. Medical Record Linkage. Mortality. 


\section{REFERÊNCIAS}

1 - Mock C, Lormand JD, Goosen J, Joshipura M, Peden M. Guidelines for essential trauma care. Geneva: World Health Organization; 2004.

2 - Brasil. Ministério da Saúde. Portaria MS/GM nº 737 de 16 de maio de 2001. Política Nacional de Redução da Morbimortalidade por Acidentes e Violência. Diário Oficial da União, $n^{\circ}$ 96, seção 1E, de 18 de maio de 2001. Brasília; 2001.

3 - Brasil. Ministério da Saúde. Secretaria Executiva. Datasus, 2007. Disponível em: http://www.datasus.gov.br. Acesso em 4 de junho de 2007.

4 - Maier RV, Rhodes M. Trauma performance improvement. In: Rivara FP et al., editors. Injury control: a guide to research and program evaluation. New York: Cambridge University Press; 2001. p. 236-49.

5 - American College of Surgeons. Resources for Optimal Care of the Injured Patient: 2006. Chicago: Committee on Trauma, American College of Surgeons; 2006.

6 - Coimbra RSM, Rasslan S. Controle de qualidade nos serviços de trauma. In: Freire E. Trauma - a doença dos séculos. Rio de Janeiro: Editora Atheneu; 2001. p. 557-61.

7 - Hoyt DB, Coimbra R. Trauma systems. Surg Clin North Am 2007; 87: 21-35.

8 - American College of Surgeons. Committee on Trauma. Ad-

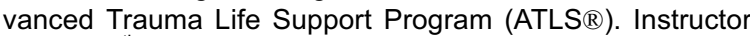
Manual. $7^{\text {th }}$. ed. Chicago: American College of Surgeons; 2004.

9 - Ali J, Adam RU, Butler AK, Chang H, Howard M, Gonsalves D. Trauma outcome improves following the Advanced Trauma Life. Support program in a developing country. J Trauma 1993; 34: 890-8.

10 - Campos Filho WO. Impacto da implementação de um programa de treinamento de recursos humanos (Advanced Trauma Life Support) sobre a qualidade da atenção prestada às vítimas de traumatismo, no âmbito hospitalar. [Dissertação de Mestrado], Ribeirão Preto: Faculdade de Medicina de Ribeirão Preto - USP; 1999.

11 - Mantovani M, Fraga GP. Escores e índices de trauma. In: Souza HP, Breigeiron R, Gabiatti G. Cirurgia do trauma: condutas diagnósticas e terapêuticas. São Paulo: Editora Atheneu; 2003. p. 19-37.

12 - Fraga GP. Índices de trauma em pacientes submetidos à laparotomia. [Tese de Doutorado], Campinas: Faculdade de Ciências Médicas - UNICAMP; 2004

13 - Champion HR, Copes WS, Sacco WJ, Lawnick MM, Keast SL, Bain LW et al. The Major Trauma Outcome Study: establishing national norms for trauma care. J Trauma 1990; 30: 1356-65.

14 - American College of Surgeons. Committee on Trauma. Consultation for trauma systems. Chicago: AC S; 1998.

15 - Guidelines for trauma care systems. American College of Emergency Physicians. Ann Emerg Med 1987; 16: 459-63.

16 - Nathens AB, Jurkovich GJ, Rivara FP, Maier RV. Effectiveness of state trauma systems in reducing injury-related mortality: a national evaluation. J Trauma 2000; 48: 25-31.
17 - Biffl WL, Harrington DT, Majercik SD, Starring J, Cioffi WG. The evolution of trauma care at a level I Trauma Center. J Am Coll Surg 2005; 200: 922-9.

18 - Celso B, Tepas J, Langland-Orban B, Pracht E, Papa L, Lottenberg $\mathrm{L}$, et al. A systematic review and meta-analysis comparing outcome of severely injured patients treated in trauma centers following the establishment of trauma systems. J Trauma 2006; 60: 371-8.

19 - National Trauma Data Bank®. Disponível em: http:// www.facs.org/trauma/ntdb.html ou http://www.ntdb.org. Acesso em 4 de junho de 2007.

20 - McLellan BA. A Canadian National Trauma Registry: the time is now. J Trauma 1997; 42: 763-8.

21 - Canadian Institute for Health Information. National Trauma Registry. 2005 Injury hospitalizations highlights report. Disponível em: http://www.cihi.ca/ntr. Acesso em 4 de junho de 2007.

22 - Cameron PA, Gabbe BJ, McNeil JJ, Finch CF, Smith KL, Cooper DJ, et al. The trauma registry as a statewide quality improvement tool. J Trauma 2005; 59: 1469-76.

23 - Di Bartolomeo S, Nardi G, Sanson G, Gordini G, Michelutto V, Ciminello $\mathrm{M}$, et al. The first Italian trauma registry of national relevance: methodology and initial results. Eur J Emerg Med 2006; 13: 197-203.

24 - Mock CN, Jurkovich GJ, nii-Amon-Kotei D, Arreola-Risa C, Maier RV. Trauma mortality patterns in three nations at different economic levels: implications for global trauma system development. J Trauma 1998; 44: 804-14.

25 - Hlaing T, Hollister L, Aaland M. Trauma registry data validation: essential for quality trauma care. J Trauma 2006; 61: 1400-7.

26 - Mock C, Nguyen S, Quansah R, Arreola-Risa C, Viradia R, Joshipura M. Evaluation of Trauma Care capabilities in four countries using the WHO-IATSIC Guidelines for Essential Trauma Care. World J Surg 2006; 30: 946-56.

27 - Mock C, Joshipura M, Quansah R, Arreola-Risa C. Advancing injury prevention and trauma care in North America and globally. Surg Clin North Am 2007; 87: 1-19.

28 - Scarpelini S, Andrade JI, Passos ADC. The TRISS method applied to the victims of traffic accidents attended at a tertiary level emergency hospital in a developing country. Injury 2006; 37: 72-7.

29 - Martin BT, Fallon Jr. WF, Palmieri PA, Tomas ER, Breedlove L. Autopsy data in the peer review process improves outcomes analysis. J Trauma 2007; 62: 69-73.

30 - Moura A. Trauma mobiliza médicos. Rev APM 2006 (565): 16-20.

Recebido em 26/06/2007

Aprovado em 27/09/2007 